\title{
Factors Influencing the Uptake of Intermittent Preventive Treatment for Malaria in Pregnancy: Evidence from Bungoma East District, Kenya
}

\author{
Angela Chepkemoi Ng'etich Mutulei* \\ Kenya Red Cross Society Department of Health and Social services Nairobi Kenya \\ *Corresponding author: memongetich@yahoo.com
}

Received May 07, 2013; Revised May 30, 2013; Accepted May 31, 2013

\begin{abstract}
Malaria infection in pregnancy is highly risky for the mother and her fetus. Intermittent Preventive Treatment in pregnancy (IPTp) with sulfadoxine-pyrimethamine is a key intervention for malaria prevention during pregnancy. Bungoma East District falls within the malaria-endemic zone of Kenya, with an infection risk class of $20 \%$ or higher. Even though malaria accounts for about $41 \%$ of maternal deaths in the District, $\mathrm{IPTp}_{2}$ coverage remains below the Roll Back Malaria target. The objective of this study was to identify and document factors influencing the $\mathrm{IPTp}_{2}+$ uptake. To achieve this, I applied the cross-sectional survey design to guide the research process. I sourced data from 278 women who were at the $24^{\text {th }}$ week or more of gestation, aged between 15 and 49 years, had not experienced malaria during the pregnancy and were not on cotrimoxazole prophylaxis. Quantitative analysis techniques included one-way analysis of variance, cross-tabulations with Chi Square statistic and binary logistic regression. The study found that women starting antenatal visits in the second month had about 10.5 times the odds of taking $\mathrm{IPTp}_{2}+$ as those initiating antenatal attendance in the sixth month of pregnancy. Again, women who mentioned health facilities as the main source of information were about 12.7 times as likely to take $\mathrm{IPTp}_{2}+$ as who relied on community health worker. Women who received support from their partners towards antenatal visits had about 8.2 times the odds of taking IPTp ${ }_{2}+$ as those who did not receive partner support. Furthermore, women dwelling within a distance of less than one hour's walk had about 6.4 times the odds of taking $\mathrm{IPTp}_{2}+$ as those dwelling within a distance of more than 4 hour's walk. Based on the findings, I recommend the need for better programming and content of health education sessions at the clinic; better staffing of health facilities to reduce workload and improve service quality; training health workers on customer care and sensitization of men through the media, work place paternal programs and peer education for a more active role.
\end{abstract}

Keywords: intermittent preventive treatment, service uptake, pregnancy, malaria prevention, malaria control

\section{Introduction}

Malaria remains a key public health issue globally, with about 3.3 billion people being at risk of infection by the end of 2012. The annual world malaria reports consistently show that Africa bears the heaviest burden and the highest risk of malaria infection. In this regard, about $80 \%$ of the reported malaria cases and about $90 \%$ of the reported deaths occur in Africa, with children below five years and pregnant women living in malaria-endemic regions suffering the most [1,2,3]. Although five species of parasites cause malaria, Plasmodium falciparum accounts for about 90 to $98 \%$ of all infections in the African region. The anopheles female mosquito is the vector responsible for the transmission of $P$. falciparum parasite to humans through its bite [1,2,3].

In Kenya, malaria is the leading cause of morbidity and mortality with about $74 \%$ of the population being at risk of infection [4]. The country has four malaria epidemiological zones based on the transmission risk, namely, endemic, highland epidemic prone, arid seasonal and low risk. Although about $29 \%$ of the population resides in malaria-endemic zones, seasonal epidemics of malaria in the highland and arid zones confer negligible immunity; thus, making the native population vulnerable to malaria [5]. In public health facilities, clinically diagnosed malaria accounts for about one-third of outpatient consultations, 19\% of hospital admissions and 3-5\% of inpatient deaths [5]. Malaria is not only a public health challenge, but also an impediment to economic productivity at the household, community and national levels. In view of this, the Government of Kenya (GoK) considers malaria control a priority investment, necessary for the realization of the Kenya Vision 2030 [5].

The National Malaria Strategy (NMS) and the third National Health Sector Strategic Plan 2012-2017 are the key policy instruments guiding malaria control activities in Kenya. The NMS outlines malaria control activities based on the epidemiological patterns in the country, with the aim of achieving national and international targets [5]. In this regards, the core interventions adopted in Kenya include vector control-using insecticide-treated nets (ITNs) and indoor residual spraying (IRS); case management using Artemisinin-based combination therapies (ACTs) 
and improved laboratory diagnosis; management of malaria in pregnancy; epidemic preparedness and response; as well as information, education and communication (IEC) for behavior change [4,5].

\subsection{Malaria Prevention in Pregnancy}

Globally, about 50 million women become pregnant each year, of which more than one-half reside in Africa, where the risk of malaria infection is highest [2]. Malaria infection during pregnancy is highly risky for the mother and her fetus, particularly due to low levels of immunity. Estimations show that malaria accounts for about 10,000 maternal deaths and about 200,000 fetal and infant deaths annually, with severe malarial anaemia contributing to more than one-half of reported deaths [2]. Malaria in pregnancy accounts for 5 to $12 \%$ of all low-weight births [6]. In low risk zones, episodes of severe malaria significantly associates with stillbirths, spontaneous abortion, premature delivery and maternal death $[7,8]$. However, in high-risk areas, women are susceptible to asymptomatic infection, with potential results being maternal anaemia and placental parasitaemia. Both situations are conducive for low birth weight and subsequently, infant mortality [6,8].

Intermittent Preventive Treatment in pregnancy (IPTp) is one of the key interventions recommended by WHO to bolster the prevention of asymptomatic infections among pregnant women living in moderate to high-risk regions [8]. In Kenya, the Government's policy on IPTp states that all pregnant women living in malaria-endemic zones should receive sulfadoxine-pyrimethamine (SP) for prevention of malaria during pregnancy. In this regard, all pregnant women should receive the first dose of three tablets $\left(\mathrm{IPTp}_{1}\right)$, which providers administer under their direct observation at the antenatal care (ANC) facilities within the $16^{\text {th }}$ week of gestation. Recipients of IPTp $_{1}$ should access subsequent doses during each of the scheduled monthly visits to ANC facilities [4]. Hence, all pregnant women should access the second dose (IPTp 2$)$ within the $20^{\text {th }}$ week of pregnancy. WHO recommends a minimum of $\mathrm{IPT}_{2}$ protection against malaria for women residing in high risk regions [9].

The African Summit on Roll Back Malaria, which took place in April 2000 set the IPTp 2 coverage target at $60 \%$ by 2005 , while the global target stands at $80 \%$ by 2010 [9]. In Kenya, the NMS 2009-2017 set the IPTp 2 coverage target at $80 \%$. However, various studies suggest that the uptake of IPTp in the Sub-Sahara African countries remains below the international targets. In Kenya, for instance, the 2008/09 Demographic and Health Survey (KDHS) report indicate that only $15.1 \%$ of the participants received two or more doses of SP tablets [4], while the Malaria Indicator Survey of 2010 recorded a national $\mathrm{IPTp}_{2}$ coverage of $25.7 \%$ [10].

\subsection{Factors Influencing the Uptake of Intermittent Preventive Treatment}

The IPTp uptake is a subject that has attracted many empirical investigations, at the community, national and regional levels, particularly in Africa. In Kenya, for instance, [11] found that personal attributes such as marital status and education level influenced the IPTp uptake. In this regard, the study noted that the IPTp uptake was significantly higher among participants with university education than those reporting primary education. In Uganda, [12] noted that lack of post primary education significantly associated with the failure of pregnant women to access IPTp I $_{1}$ Contrastingly, in Tanzania, [13] reported lack of significant association between the uptake of $\mathrm{IPTp}_{2}$ and factors such as age, marital status and education level. The study noted that women having less than primary education were about three times more likely to default in subsequent ANC visits than those reporting secondary education or higher.

In Senegal, [14] reported that the timing of IPTp directly linked to the onset of ANC visits. On the same note, [15] found that delayed attendance of ANC contributed to non-completion of IPTp doses. In this regard, $45 \%$ of the participants initiated ANC attendance in the third trimester and only $23.7 \%$ received $\mathrm{IPTp}_{2}$. The timing of first visits to ANC clinics also influences the IPTp uptake. In this regard, [16] found that even though $48 \%$ of the participants started ANC visits before the $16^{\text {th }}$ week of pregnancy, up to $86 \%$ of this lot did not receive IPTp$_{1}$ because the gestation period was below the recommended 16 weeks. Those who did not receive proper explanation of this policy requirement were discouraged and failed to turn up for subsequent appointments.

Furthermore, IPTp uptake significantly associated with distance to ANC facilities, domestic chores and absence of health issues during pregnancy [15], as well as gravidity [16]. Hence, primigravid women were more likely to attend ANC in time for IPTp doses than multigravid women. The study noted that first-time pregnant women sought medical assistance earlier than experienced mothers, to pacify anxiety arising due to sudden physiological changes.

Previous studies have also indicated that the IPTp uptake significantly associates with women's knowledge regarding IPTp. In their study, [17] found that only $21 \%$ of the participants had received information about IPTp, of which only $31.5 \%$ knew the recommended drugs for IPTp and only $4.5 \%$ knew the recommended dose. The study found that over $60 \%$ of the participants did not receive health education on IPTp, resulting to low IPTp coverage [17]. Other studies such as [18] and [19] also confirm that knowledge about IPTp remains low among pregnant women in developing countries, which suggests that ANC facilities are not doing enough on sensitization. However, [15] links the knowledge gap to late and inconsistent ANC attendance.

The quality of services at health facilities is also a key factor influencing the IPTp uptake. For instance, a study conducted by [14] reports that most public health facilities in Ghana (94.1\%) provided IPTp services. However, up to $27 \%$ of the facilities had experienced SP stock-outs over the preceding six months period, which significantly undermined the delivery of IPTp services. On the same note, [20] as well as [21] found that recurrent SP stockouts, inconsistent supply of clean drinking water and inadequacy of clean cups were some of the facility factors influencing the IPTp uptake. Furthermore, [22] report that negative attitudes towards late ANC attendees discouraged subsequent visits for IPTp services. Besides, [23] noted that prolonged waiting time, mishandling by nurses and 
lack of diagnostic facilities discouraged women from seeking ANC services.

Bungoma East District falls within Kenya's malariaendemic zones. Anecdotal information from the Health Management Information Systems suggest that the $\mathrm{IPTp}_{2}$ coverage between July 2009 and June 2010 averaged at 47.5\%, while the District Annual Operational Plan 20102011 places the $\mathrm{IPTp}_{2}$ coverage at 35\% [24]. Despite the inconsistency, available information suggests that the $\mathrm{IPTp}_{2}$ coverage in the District remains below the national and international targets, set at $80 \%$ by 2010 [4,5,9]. The gap portends a lot of harm for pregnant women and their unborn babies in the District and impedes the realization of national development goals. A review of pertinent literature revealed a paucity of information regarding factors influencing the IPTp uptake in Bungoma East District, as no systematic academic process had ever explored the subject. Consequently, this study sought to identify and document factors influencing the IPTp uptake to justify the need for appropriate interventions, which would reduce the gap in IPTp coverage and improve malaria prevention in pregnancy.

\subsection{Theoretical Framework}

This study anchors on the Health Belief Model (HBM) and Health Promotion Model (HPM) of Nola Pender. HBM emerged in the 1950s to explain why medical screening programs offered by the US Public Health Service were not always successful [25]. The model holds that perceptions about a disease and strategies available to decrease its occurrence determine health behavior [25]. The original HBM consisted of four constructs, namely: perceived seriousness, perceived susceptibility, perceived benefits and perceived barriers. However, HBM scholars later improved the accuracy of the model by including three new constructs, namely, cues to action, modifying factors and self-efficacy [26].

Perceived seriousness signifies an individual's belief about the severity of a disease. While the perception of seriousness arises from medical information or knowledge, it may also come from beliefs a person holds about the difficulties a disease would create in his or her life [27]. For instance, an individual may view that flue is less serious than malaria infection, because, while flue would require a few days to clear, malaria requires a longer period and finances to treat. Hence, one is likely to change behavior faster when facing the risk of malaria than flue.

Perceived susceptibility is one of the powerful perceptions causing the adoption of prescribed health behaviors. The greater the perceived risk of infection, the higher the chance of behavior change. For instance, perceived susceptibility is what drives pregnant women to use Long Lasting Impregnated Nets (ILLNs) to decrease their susceptibility to malaria infection. When people perceive that they are at risk of infection, they are likely to prevent it from happening by adopting prescribed measures. However, when people believe that they are not at risk, the adoption of preventive behaviors remains low [28].

Perceived benefits refer to an individual's opinion about the value of a new behavior in decreasing the risk of contracting an infection. People tend to adopt healthier behaviors when they believe such new behaviors will decrease their chances of developing a disease. Perceived benefits play an important role in the adoption of preventive behaviors, such as consistent use of LLINs, regular visits to ANC clinics for preventive medication. Figure 1 summarizes the linkage between different constructs of the model.

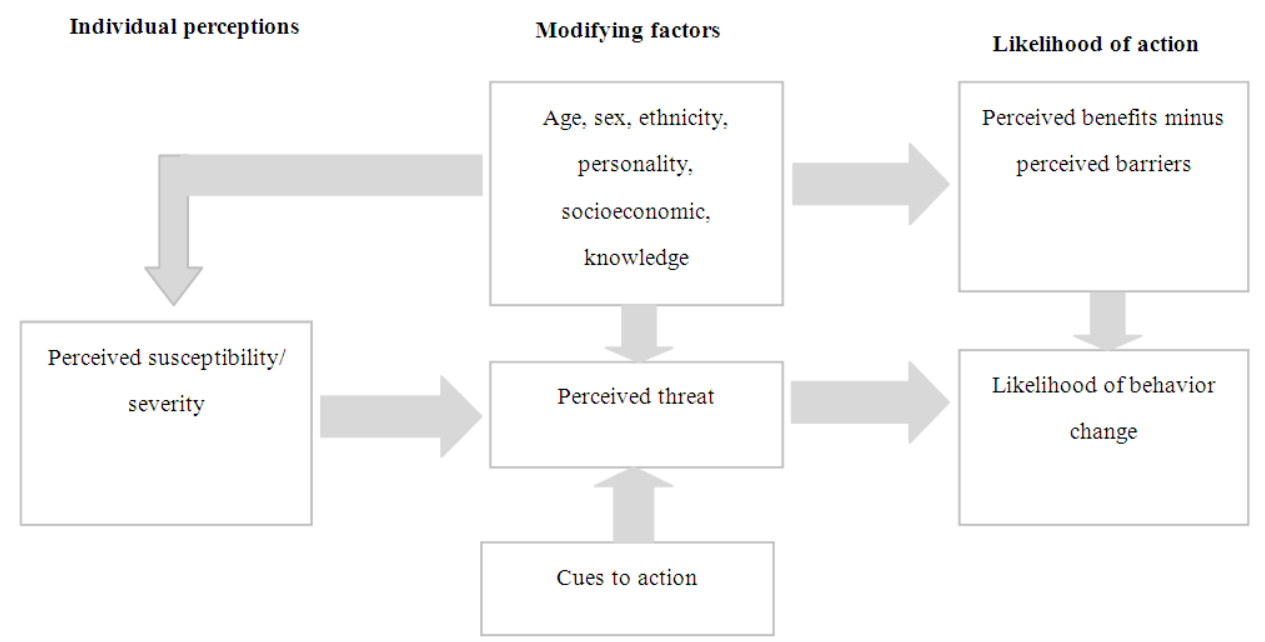

Figure 1. The Health Belief Model [30]

Perceived barriers address the issue of obstacles to behavior change. This is an individual's own evaluation of the obstacles to the adoption of preventive behaviors. As noted by [29], of all the constructs, perceived barriers are the most significant in determining health behavior change. To start visiting ANC clinics during the early stages of pregnancy, a pregnant woman needs to believe that the benefits of such visits outweigh the consequences of not visiting the facilities. This bolsters the will to overcome barriers and encourages one to seek antenatal care services.
Barriers to behavior change may also include financial constraints, stigma, embarrassment or fear of pain, among others.

Modifying variables such as gender, age, religion, marital status, parity, gravidity, education level, occupation, past experiences and average income, among others influence the strength of the original theoretical constructs. These characteristics influence personal perceptions regarding a particular behavior and the likelihood of behavior adoption [31]. Cues to action, 
including events or people may move an individual to change behavior. Examples include illness of a family member, partner's opinion about antenatal care, peer influence or health warnings in the media, associated with non-conformity [31].

Self-efficacy refers to one's own belief on his/her ability to do something [32]. Generally, people do not try out new things unless they believe they can do it. If someone believes that a new behavior is useful, but does not think he or she is capable of doing it; chances are high that such new behaviors may not be tried. For instance, if a pregnant woman believes that medication during pregnancy may harm her fetus, then the uptake of IPTp as a new behavior for reducing susceptibility to malaria infection may be constrained.

The HBM is however, faulted for inadequate consideration of attitude in behavior change. Attitude is a subjective attribute, synonymous to liking or disliking service quality, service providers or health facilities. Socialization, pertinent information, religion and education level, among others shape attitudes. Even though a particular behavior may be effective in reducing vulnerability to malaria infection, its adoption largely depends of an individual's attitude. Nevertheless, HBM is applauded for taking into consideration the effect of personal attributes, including demographic and socioeconomic factors [26], which make the model most appropriate for this study.

Nola Pender, a Professor of Nursing at the University of Michigan developed the Health Promotion Model (HPM) in 1982 to guide research activities explaining and predicting health behavior. The model derived inspiration from HBM, which came into existence about two and half decades earlier. Unlike HBM, whose theoretical thrust centers on the notion that fear of infection is the key factor motivating individuals to seek preventive interventions, HPM provides a broader picture of health and determinants of health behavior. The model posits that good health does not merely mean the absence of disease; rather, it includes measures taken to promote good health, instill a sense of self-care responsibility, access early detection and prevent illness [33,34,35].

The model assumes that people: attempt to create optimal living conditions, have the ability to complete a self-assessment and know their strengths, value growth, seek to regulate their behavior, as well as interact and transform their environment. Besides, the model assumes that health workers are an integral part of the community and that people have the ability to change their behavior as necessary [36].

HPM's concepts anchor on two theories - the expectancy value theory and the social cognitive theory. The expectancy value theory stems from the idea that the course of action is likely to lead to desired outcome, which is likely to be of personal value [37]. The social cognitive theory centers on the concept of self-efficacy, which is an individual's perception about their ability to carry out a particular behavior. The stronger it is, the higher the likelihood that behavior change will be achieved [34,37].

The major concepts constituting HPM are three and they include individual characteristics and experiences, behavior specific cognitions and effect, as well as behavior outcome [38]. Individuals seek to utilize past experience and behavior to assess the possibility of change. In this regard, women's decision to take IPTp2+ depends on their experiences with IPTp1 in terms of general weakness of the body, the waiting time at the facility and providers' attitude, among other variables. Personal characteristics such as educational attainment, partner support and income level, among others, influence an individual's health behavior decisions. Personal attributes also help individuals to identify patterns that maybe beneficial, strengths and weaknesses and is a great way to create a platform to formulate a plan of action [38].

Behavior specific cognitions and affect concept is extrapolated from situational influences. Due to environmental influences such as community misconceptions about SP tablets, economic constraints, long distance to health facilities and cultural beliefs, among others, each plan considers possible reasons for failure in behavior change or factors that may impede behavior change. This plan will clearly outline the benefit of changing one's behavior and the steps necessary to attain such a goal [38].

Behavioral outcome, measures the viability of the plan of action and resources available versus resources to be attained. In this tailored plan, health workers should seek to empower individuals with information necessary to achieve success, good health and increased capacity for self-care, thereby highlighting the behavioral outcome of health promotion. Through health promotion, pregnant women are empowered with necessary knowledge and skills on malaria prevention, signs and symptoms and the importance of the IPTp intervention. Besides, through health promotion, providers instill self-care responsibilities, which may go a long way in improving IPT $_{2}+$ uptake. HPM influenced the choice of methods used in this study, interpretation and discussion of the findings [38].

\section{Methodology}

Kenya has four malaria eco-epidemiological zones, namely, the highland epidemic-prone, malaria-endemic, seasonal malaria transmission and low-risk malaria areas. The highland epidemic-prone zone, covering highlands to the west of the Rift Valley, has a risk class of 5\% to less than 20\%; the malaria-endemic zone, which covers the Lake Victoria, Western and Coastal regions, has a risk class of $20 \%$ or higher. The seasonal malaria transmission zone, including parts of Rift Valley, Eastern and North Eastern regions, has a risk class of less than $5 \%$, while the low-risk zone, which includes Nairobi and Central regions, has a risk class of less than $0.1 \%$ [10].

\subsection{The Study Area}

Bungoma East District falls within the malaria-endemic zone on the eco-epidemiological map of Kenya. According to the 2009 Population and Housing Census, the District had a population of 257,611 of which 61,827 were women in the reproductive age [39]. The Health Management Information Systems (HMIS) show that between the months of July and December 2010, the District recorded about 704 maternal deaths, of which malaria contributed 41\% (unpublished HMIS reports). Hence, malaria remains the single most important threat to 
pregnant women and their unborn babies. With an infection risk of $32 \%$, the District reports one of the highest infection rates in Western Province [24]. Prior to the study, I managed to access consistent malaria epidemiological data for the period of July to December 2010 from five districts located in the malaria-endemic zone. Based on this, the selection of Bungoma East District from the set of five districts was purely random.

\subsection{Design and Target Population}

I applied the cross-sectional survey design, with both quantitative and qualitative approaches to source, process and analyze the requisite information. The quantitative approach elicited quantifiable and numerical data from pregnant women, which I used to generate descriptive and inferential results. The qualitative approach targeted health service providers at the clinics. The paper presents part of the quantitative results, qualitative results have been set for publication in an upcoming manuscript on provider factors influencing IPTp uptake. The study targeted pregnant women coming to public health facilities, including dispensaries, health centres, as well as sub-district and district hospitals for routine antenatal care services. The district statistical fact sheet indicates that about 3,091 pregnant women register at the ANC clinics each year, and making an average of 2.5 ANC visits. I designated this number the population from where I drew the sample.

\subsection{Sample Size Determination}

With a finite population of 3,091 in mind, I applied Fisher's formula for sample size determination to obtain a sample $\left(n_{i}\right)$.

$$
n_{i}=\left\{\frac{\delta(1-\delta)}{\left(\frac{\alpha}{z}\right)^{2}+\delta(1-\delta) / N_{i}}\right\} * \mu_{i}
$$

Where, $n_{i}=$ effective sample size, $N_{i}=$ population: 3,091 pregnant women, $\delta=$ estimated population variance: $0.5, \alpha$ = desired precision: $0.05, Z=$ confidence level: 1.96 for 95\% on the normal distribution curve, $\mu_{i}=$ design effect: 1.024. Based on these parameters, the computation yielded an effective sample size of $349.89 \approx 350$ women. This is about $11.3 \%$ of the number of women registering at the ANC clinics each year.

\subsection{Sampling Process}

Public health facilities in Bungoma East District fall into three tiers based on the number of services offered, including level four, three and two. Level four facilities included the district and sub-district hospitals; level three facilities were health centres, while level two facilities included dispensaries. I noted that all public health facilities provided IPTp services, irrespective of the tier. Based on this information, I applied a three-stage sampling process to obtain target respondents.

At the first stage, I developed a list of public health facilities, with the assistance of the District Medical Officer of Health (DMOH). The District had 10 public health facilities, including 1 district hospital, 1 sub-district hospital, 2 health centres and 6 dispensaries. I stratified the list based on the tiers. In the strata of level four and level three facilities, I applied purposive sampling procedure to select Webuye District Hospital, Bokoli SubDistrict Hospital, as well as Webuye and Milo Health Centres. In the stratum of level two facilities, I applied stratified random procedure to select Khaoya, Mukhe and Sinokoo Dispensaries. The stratification criterion ensured proportionate inclusion of rural and urban-based dispensaries. Stratified random sampling procedure ensures a fair representation of elements whose number in the sampling frame is small [40].

At the second stage, I applied proportionate sampling procedure to determine proportionate samples for each facility based on the previous workload. In this regard, I collected information from ANC clinic registers about the number of registered clients from July 2010 to June 2011. Table 1 shows the number of clients registered at the ANC clinics in each health facilities over the reference period. To determine proportionate samples for each facility, I applied the formula stated below:

$$
n_{o}=f * s N_{O}
$$

Where $n_{o}=$ stratum sample size; $f=$ the sampling fraction $\left(n_{i} / N_{i}\right)$ and $s N_{o}=$ the stratum population. I established the average monthly ANC attendance in each facility. The quotient of the desired sample size $\left(n_{i}\right)$ and the target population $\left(N_{i}\right)$ yielded a sampling fraction $(f)$ of 0.12048. Using the sampling fraction, I computed proportionate samples for each health facility as indicated in Table 1 below.

\begin{tabular}{|c|c|c|c|c|c|}
\hline Strata & Sampled Facilities & $\begin{array}{c}\text { Registered clients } \\
\text { at ANC facility }\left(\mathrm{sN}_{0}\right)\end{array}$ & $\begin{array}{l}\text { Stratum sample } \\
\operatorname{sizes}\left(\mathrm{n}_{0}=\mathrm{f}^{*} \mathrm{sN}_{\mathrm{o}}\right)\end{array}$ & $\begin{array}{l}\text { \# of visits at the } \\
\text { ANC facility (a) }\end{array}$ & $\begin{array}{l}\text { Average monthly visits } \\
\text { at the ANC facility }(\mathrm{a} / 12)\end{array}$ \\
\hline \multirow{2}{*}{$\begin{array}{c}\text { Level } \\
4\end{array}$} & Webuye District Hospital & 643 & 77 & 1,611 & 134 \\
\hline & Bokoli Sub-District Hospital & 507 & 61 & 1,257 & 105 \\
\hline \multirow{2}{*}{$\begin{array}{l}\text { Level } \\
3\end{array}$} & Webuye Health Centre & 575 & 69 & 1,442 & 120 \\
\hline & Milo Health Centre & 351 & 42 & 878 & 73 \\
\hline \multirow{4}{*}{$\begin{array}{l}\text { Level } \\
2\end{array}$} & Khaoya Dispensary & 327 & 39 & 813 & 68 \\
\hline & Mukhe Dispensary & 239 & 29 & 592 & 49 \\
\hline & Sinokoo Dispensary & 263 & 32 & 665 & 55 \\
\hline & Total & 2,905 & 350 & 7,258 & 605 \\
\hline
\end{tabular}

Table 1. Proportionate sample sizes for each health facility

At the third stage of the sampling process, I targeted pregnant women visiting ANC clinics. In this regard, I applied systematic random sampling procedure to identify participants. To determine the sampling interval, I sourced information from clinic registers about the number of
ANC visits recorded between July 2010 and June 2011 for each facility, as presented in Table 1. Using this information, I computed monthly averages because I scheduled data collection to last for one month. I then computed the quotient of average monthly visits $(a / 12)$ 
and stratum sample sizes $\left(n_{o}\right)$, which gave us an interval of $1.7281 \approx 2$. Based on this, I sampled every second woman exiting ANC facilities. Sampled women were screened and those meeting the inclusion criteria taken through verbal consenting process before interviews.

\subsection{Inclusion and Exclusion Criteria}

I included pregnant women aged 15 to 49 years, who had gestated for at least six months (24 weeks) and consented to participate in the study voluntarily. WHO recommends that pregnant women should access $\mathrm{IPTp}_{2}$ within the $20^{\text {th }}$ week of pregnancy. By pegging the inclusion criterion at $24^{\text {th }}$ week, I provided an allowance for late clinic attendees, who may have not accessed the second dose within the recommended time. However, I excluded women who were on cotrimoxazole prophylaxis and those who had received treatment for malaria during the pregnancy.

\subsection{Data Collection Instrument}

We applied a standard survey questionnaire to source information through exit interviews with women. Among other information, the questionnaire captured data on socio-demographic and economic attributes, knowledge of IPTp, ANC clinic attendance, health education attendance, IPTp uptake as well as perceptions about various aspects of the IPTp intervention. We translated the questionnaire into Swahili language to facilitate communication, ease its implementation and standardize responses. We pre-tested the questionnaire in July 2011 at Khaoya Dispensary using 32 respondents, with each member of the team conducting four interviews. We applied the split-half technique to estimate reliability of the instrument. The process yielded a Cronbach's Alpha value of 0.7448 for part 1 and 0.7418 for part 2, which suggests a satisfactory level of internal consistency [41,42].

\subsection{Data Collection Process}

I engaged seven research assistants on merit to support the data collection process. All the assistants were holding bachelors' degrees in social sciences and had participated in at least two research projects before. I trained them for three days to refresh the basics of data sourcing skills, agree on approaches and understand requirements of the questionnaire. I assigned the research assistants to specific health facilities and I facilitated them to visit the facilities on clinic days each week. I coordinated data collection activities, supervised the assistants, managed the research process and controlled data quality. We collected data in August 2011 and the process yielded a net of 278 (79.4\%) successful interviews, with good quality data. I excluded from analysis incomplete questionnaires and those containing inconsistent or missing information.

\subsection{Data Processing and Analysis}

Data processing involved coding open-ended and multiple responses, digitalization and cleaning for misplaced codes. For some variables, I transformed the scale of measurement to suit the chosen analysis techniques. Quantitative analysis techniques included cross-tabulations with Chi Square statistic and independent samples t-test for interval-scaled variables. I further applied binary logistic regression to determine the odds of women receiving $\mathrm{IPTp}_{2}$ based on their perceptions. Binary logistic regression predicts the proportion of variance in a dichotomous variable from a set of independent variables (Wuensch, 2006). The predicted variable takes the value 1 with a probability of success $\theta$, or the value 0 with a probability of failure $1-\theta$. The dependent variable in this study was the $\mathrm{IPT}_{2}$ uptake, with only two possible values - less than IPTp 2 or IPTp $\mathrm{IP}_{2}+$. The model takes the form:

$$
\begin{aligned}
\operatorname{Logit}[\theta(Y)] & =\log \left[\frac{\theta(Y)}{1-\theta(Y)}\right] \\
& =\alpha+\beta_{1} X_{1}+\beta_{2} X_{2}+\beta_{3} X_{3} \cdots+\beta_{i} X_{i}+\varepsilon_{i}
\end{aligned}
$$

Where $Y=$ dependent variable (IPTp $\mathrm{p}_{2}$ uptake); $\theta(Y)=$ the probability that a pregnant woman had received the $\mathrm{IPTp}_{2}+; 1-\theta(Y)=$ the probability that a pregnant woman had received less than IPTp $; \alpha=$ the constant term of the equation; $\beta_{1}, \beta_{2} \ldots \beta_{i}=$ partial regression co-efficients associated with independent variables; $X_{1}, X_{2} \ldots X_{i}=$ independent variables and $\varepsilon=$ the error term. The logistic regression generated beta co-efficients $(\beta)$, odds ratios $[\operatorname{Exp}(\beta)]$ and -2 Log Likelihood statistic, HosmerLemeshow test of goodness-of-fit and Nagelkerke's $\mathrm{R}^{2}$. Detailed description of the design and approaches that we used in this study are available in following publications $[40,43,44,45]$.

\subsection{Ethical Considerations}

The Institutional Research and Ethics Committee (IREC) of Moi Teaching and Referral Hospital and the School of Medicine, Moi University approved the study in July 2011. Other institutions that also approved the study included the Ministry of Education, Science and Technology and the Ministry of Health. We observed three universal ethical principles, including respect for participants, beneficence and justice. All participants were consented by fully explaining purpose of the study, potential benefits, and the fact that their participation was voluntary. We also informed participants about their right to withdraw consent at any time during the process without a penalty. Since, the survey had no clinical components; the risks involved were minimal.

Furthermore, we kept the information sourced confidential and ensured the confidentiality of interviews, at least audibly. We avoided information on personal identifiers, assured participants that we would use the information for research purposes only, with access limited to the investigators only. We assured participants that we would share the report with the Government, Moi University, Moi Teaching and Referral Hospital and other stakeholders to support decisions aimed at making the IPTp intervention more responsive and sensitive to perceptions held by pregnant women. In this regard, we adhered to the provisions of 45 CFR 46.101 (b) (2). Lastly, we accorded equal opportunity to participants who consented to participate in the study.

\section{Results}

The study covered 278 participants, of which 110 (39.6\%) were sampled from the district and sub-district 
hospitals, 88 (31.7\%) were interviewed from health centres, and 80 (28.8\%) sampled from dispensaries. We requested participants to indicate if they had received any medicine from the facilities to prevent malaria infection and the number of times they had received such medication. The results indicated that out of 278 participants, 157 (56.5\%) had accessed two or more doses $\left(\mathrm{IPTp}_{2}+\right.$ ), while 121 (43.5\%) had received less than two doses $\left(<\mathrm{IPTp}_{2}\right)$.

The onset of antenatal care (ANC) visits is a key factor in the uptake of IPTp, as shown by previous studies [14, 15]. The results of this study indicated that 127 (45.7\%) participants started their ANC visits within the fourth month of pregnancy, 65 (23.4\%) started within the fifth month, while 48 (17.3\%) indicated the third month. Overall, 209 (75.2\%) participants started their ANC visits within the second trimester, while 5 (1.8\%) initiated their attendance in the third trimester. On average, participants who had received $\mathrm{IPTp}_{2}+$ started their ANC visits at 3.79 months ( $\mathrm{SD}=0.877$ ), while those who accessed less than $\mathrm{IPTp}_{2}$ initiated clinic attendance a little later at 4.55 months $(\mathrm{SD}=1.024)$.
Using one-away Analysis of Variance (ANOVA), the analysis obtained a computed $\mathrm{F}_{(1,276)}$ statistic of 44.747 and a $\rho$-value of 0.000 , which was significant at 0.01 error margin. This confirms that participants who had received IPTp $_{2}+$ started their ANC visits significantly earlier than those who had received less than $\mathrm{IPTp}_{2}$. This suggests up to $99 \%$ chance than the two groups were significantly different in terms of the onset of ANC visits; hence, the uptake of IPTp significantly correlated with the onset of ANC visits.

\subsection{Background Profile of Participants}

The results indicated that participants were aged between 16 and 44 years. Table 2 shows that 49 (40.5\%) participants who had received less than $\mathrm{IPTp}_{2}$ and 51 (32.5\%) who had received IPTp $\mathrm{P}_{2}$ + were aged between 25 and 29 years; while 27 (22.3\%) who had obtained less than $\mathrm{IPTp}_{2}$ and 43 (27.4\%) who had accessed IPTp 2 + were in the 20 to 24 years age bracket. The summary of Chi Square Tests shows lack of a significant association between the uptake of IPTp and participant's age.

Table 2. Participants' background attributes

\begin{tabular}{|c|c|c|c|c|c|c|c|}
\hline \multirow{2}{*}{ Background attributes } & \multicolumn{2}{|c|}{$<\mathrm{IPTp}_{2}$} & \multicolumn{2}{|c|}{$\mathrm{IPTp}_{2}+$} & \multicolumn{3}{|c|}{ Summary of Chi Square Tests } \\
\hline & Frequency & Percent & Frequency & Percent & Computed $\chi^{2}$ & $d f$ & $\rho$-value \\
\hline Age & & & & & & & \\
\hline$<20 \mathrm{yrs}$ & 7 & 5.8 & 14 & 8.9 & & & \\
\hline $20-24 \mathrm{yrs}$ & 27 & 22.3 & 43 & 27.4 & & & \\
\hline $25-29$ yrs & 49 & 40.5 & 51 & 32.5 & 3.383 & 5 & 0.641 \\
\hline $30-34$ yrs & 25 & 20.7 & 34 & 21.7 & & & \\
\hline $35-39$ yrs & 8 & 6.6 & 11 & 7.0 & & & \\
\hline $40-44$ yrs & 5 & 4.1 & 4 & 2.5 & & & \\
\hline Total & 121 & 100.0 & 157 & 100.0 & & & \\
\hline Religion & & & & & & & \\
\hline Catholic & 41 & 33.9 & 63 & 40.1 & & & \\
\hline Protestant & 77 & 63.6 & 84 & 53.5 & 4.980 & 3 & 0.173 \\
\hline Muslim & 0 & 0.0 & 3 & 1.9 & & & \\
\hline Others & 3 & 2.5 & 7 & 4.5 & & & \\
\hline Total & 121 & 100.0 & 157 & 100.0 & & & \\
\hline
\end{tabular}

$*, * *, * * *$ represents significance at $0.1,0.05$ and 0.01 error margins, respectively

Table 3. Participants' background attributes

\begin{tabular}{|c|c|c|c|c|c|c|c|}
\hline \multirow{2}{*}{ Background attributes } & \multicolumn{2}{|c|}{$<\mathrm{IPTp}_{2}$} & \multicolumn{2}{|c|}{$\mathrm{IPTp}_{2}+$} & \multicolumn{3}{|c|}{ Summary of Chi Square Tests } \\
\hline & Frequency & Percent & Frequency & Percent & Computed $\chi^{2}$ & $d f$ & $\rho$-value \\
\hline \multicolumn{8}{|l|}{ Marital status } \\
\hline Single & 39 & 32.2 & 22 & 14.0 & & & \\
\hline Married & 74 & 61.2 & 129 & 82.2 & & & \\
\hline Separated & 5 & 4.1 & 5 & 3.2 & 17.267 & 4 & $0.002 * * *$ \\
\hline Divorced & 1 & 0.8 & 1 & 0.6 & & & \\
\hline Widowed & 2 & 1.7 & 0 & 0.0 & & & \\
\hline Total & 121 & 100.0 & 157 & 100.0 & & & \\
\hline \multicolumn{8}{|l|}{ Parity } \\
\hline Zero & 27 & 22.3 & 5 & 3.2 & & & \\
\hline 1-2 children & 49 & 40.5 & 93 & 59.2 & & & \\
\hline 3-4 children & 31 & 25.6 & 39 & 24.8 & 28.441 & 4 & $0.000 * * *$ \\
\hline 5-6 children & 10 & 8.3 & 18 & 11.5 & & & \\
\hline 7 children + & 4 & 3.3 & 2 & 1.3 & & & \\
\hline Total & 121 & 100.0 & 157 & 100.0 & & & \\
\hline \multicolumn{8}{|l|}{ Education level } \\
\hline No education & 3 & 2.5 & 1 & 0.6 & & & \\
\hline Primary & 52 & 43.0 & 54 & 34.4 & & & \\
\hline Secondary & 61 & 50.4 & 75 & 47.8 & 13.375 & 4 & $0.010^{* *}$ \\
\hline College & 5 & 4.1 & 25 & 15.9 & & & \\
\hline University & 0 & 0.0 & 2 & 1.3 & & & \\
\hline Total & 121 & 100.0 & 157 & 100.0 & & & \\
\hline
\end{tabular}

Table 2 shows that 77 (63.6\%) participants who had received less than IPTp2 and 84 (53.5\%) who had accessed IPTp2+ were protestants, while 41 (33.9\%) participants in the first group and $63(40.1 \%)$ in the second group were of the Catholic faith. However, the analysis revealed that the uptake of IPTp and religious affiliation were not significantly associated. Regarding marital status, Table 3 shows that up to 74 (61.2\%) 
participants who had received less than IPTp2 and 129 (82.2\%) who had accessed IPTp2+ were married. The analysis obtained a computed $\chi 2$ value of 17.267 , with 4 degrees of freedom and a $\rho$-value of 0.002 , suggesting up to $99 \%$ chance that the uptake of IPTp and marital status were significantly associated.

Table 3 shows that $49(40.5 \%)$ participants who accessed less than IPTp 2 and 93 (59.2\%) who had received $\mathrm{IPTp}_{2}+$ had 1 to 2 children, while those with 3 to 4 children included 31 (25.6\%) participants who obtained less than $\mathrm{IPTp}_{2}$ and 39 (24.8\%) who received $\mathrm{IPTp}_{2}+$. The summary of Chi Square results suggest up to $99 \%$ chance that the uptake of IPTp significantly associated with women's parity status.

Regarding education level, 61 (50.4\%) participants who received less than $\mathrm{IPTp}_{2}$ and 75 (47.8\%) who accessed $\mathrm{IPTp}_{2}+$ had attained some secondary education, while those with primary education included 52 (43.0\%) participants with less than $\mathrm{IPTp}_{2}$ and 54 (34.4\%) who indicated $\mathrm{IPTp}_{2}+$. The Chi Square Test suggests up to 95\% chance that the uptake of IPTp significantly associated with women's educational attainment.

Furthermore, Figure 2 shows that most participants were earning a living through farming and business. More particularly, 45 (37.2\%) participants who had received less than $\mathrm{IPTp}_{2}$ and 87 (55.4\%) who reported $\mathrm{IPTp}_{2}+$ were farmers, while $50(41.3 \%)$ who reported less than $\mathrm{IPTp}_{2}$ and $22(14.0 \%)$ who accessed $\mathrm{IPTp}_{2}+$ were engaged in informal business activities. The analysis obtained a computed $\chi^{2}$ value of 39.021 , with 5 degrees of freedom and a $\rho$-value of 0.000 , suggesting up to $99 \%$ chance that the uptake of IPTp significantly associated with women's occupation.

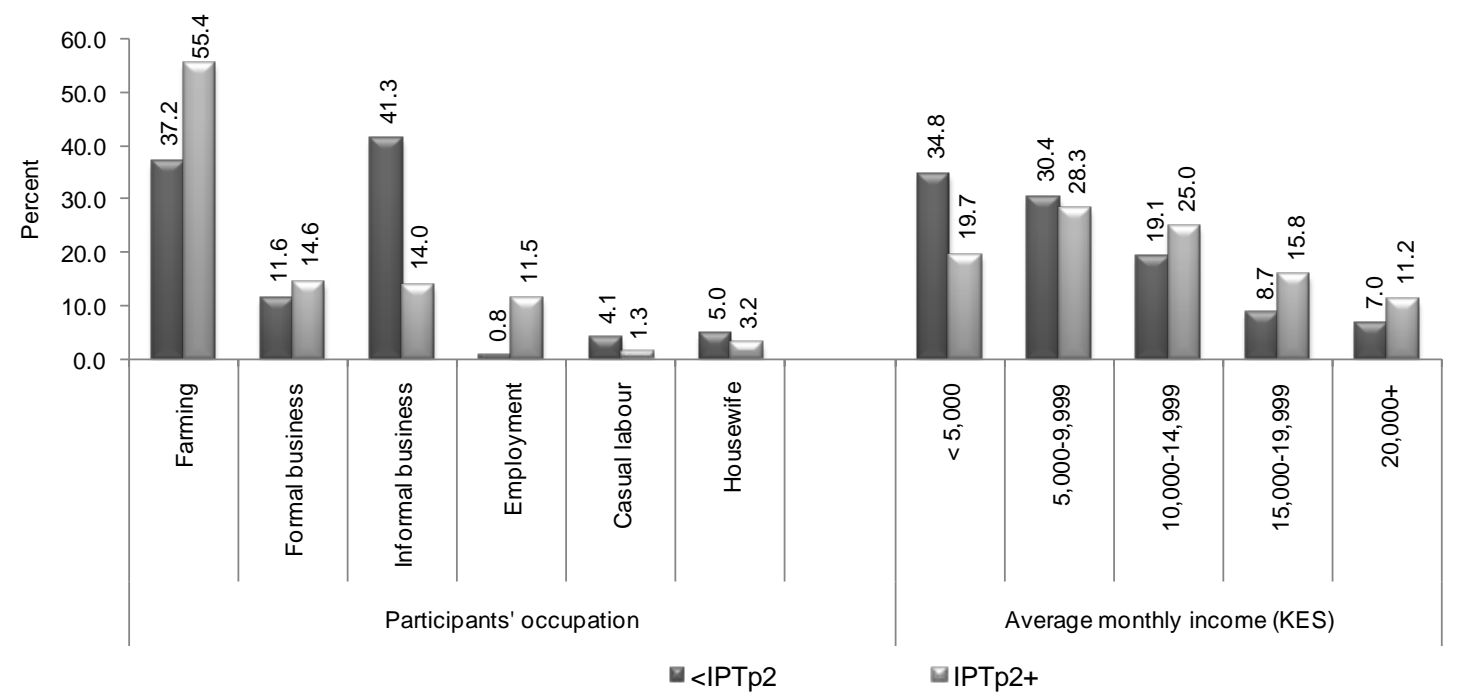

Figure 2. Occupation and average monthly income

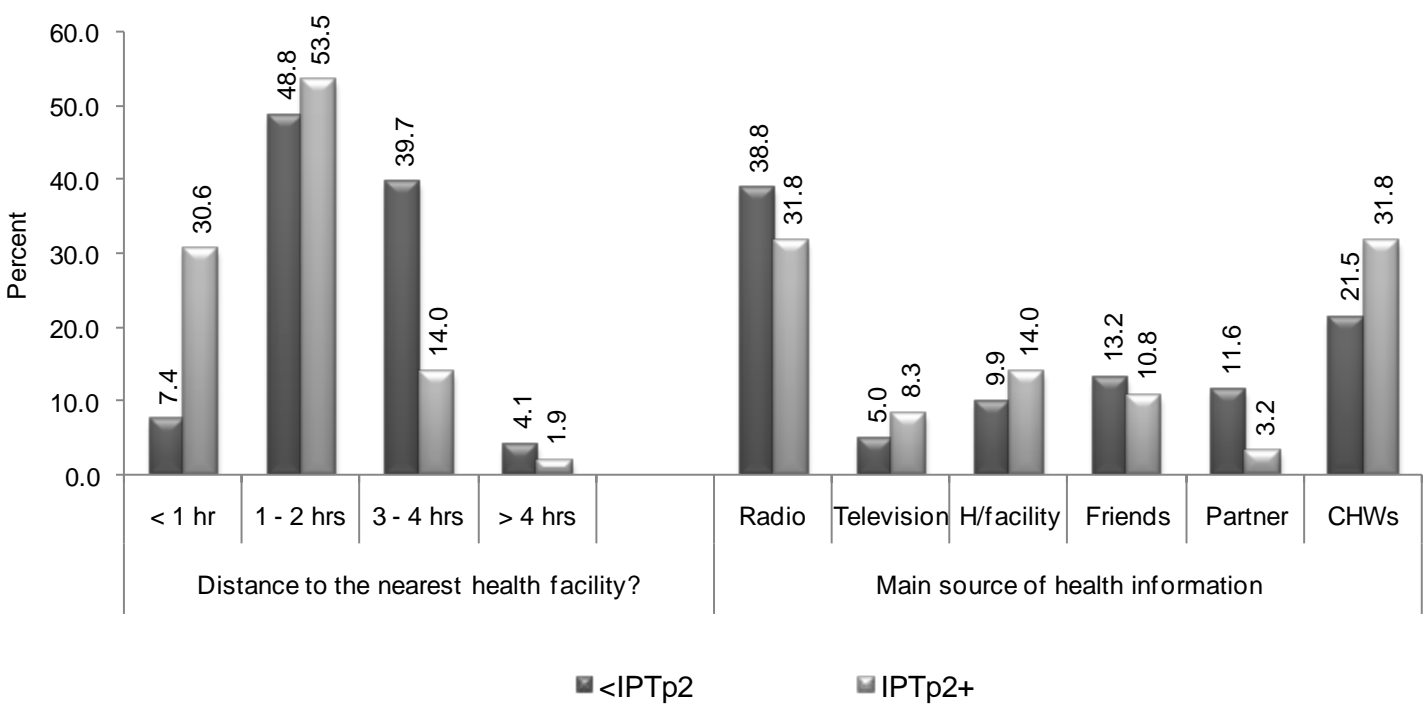

Figure 3. Duration taken to ANC facility and health information sources

We further requested participants to indicate their income, which we standardized into monthly incomes. Figure 2 shows that most participants in both groups were earning less than KES 15,000 per month. In the income bracket of KES 5,000 to 9,999 were 35 (30.4\%) participants who received less than $\mathrm{IPTp}_{2}$ and $43(28.2 \%)$ who reported having received $\mathrm{IPTp}_{2}+$. Those having incomes of below KES 5,000 included 40 (34.8\%) participants with less than $\mathrm{IPTp}_{2}$ and 30 (19.7\%) who accessed $\mathrm{IPTp}_{2}+$. The analysis obtained a computed $\chi^{2}$ value of 10.597, with 4 degrees of freedom and a $\rho$-value of 0.031 , suggesting up to $95 \%$ chance that the uptake of 
IPTp significantly associated with women's average incomes.

\subsection{Distance to ANC Facilities and Main Source of Health Information}

Figure 3 shows that most participants resided within a distance of 1 to 2 hours' walk to the nearest ANC facility. This included 59 (48.8\%) who received less than $\mathrm{IPTp}_{2}$ and 84 (53.5\%) who affirmed receiving IPTp $_{2}+$. Only 9 (7.4\%) who accessed less than $\mathrm{IPTp}_{2}$ and 48 (30.6\%) indicating $\mathrm{IPTp}_{2}+$ resided within a distance of less than an hour's walk. The results suggest up to $99 \%$ chance that the uptake of IPTp significantly associated with the distance covered to the nearest ANC facility (computed $\chi^{2}$ value $=$ $37.173, \mathrm{df}=3$ and $\rho$-value $=0.000$ ).

We further requested participants to indicate their main source of health information. The results in figure 3 indicate that out of 121 participants who received less than $\mathrm{IPTp}_{2}, 47$ (38.8\%) mentioned radio, 26 (21.5\%) cited community health workers (CHWs), while 16 (13.2\%) stated friends as the main source of health information. Among the 157 participants who had accessed $\mathrm{IPTp}_{2}+, 50$ (31.8\%) indicated radio, another 50 (31.8\%) cited CHWs, while 22 (14.0\%) mentioned health facilities as the key sources of health information. Based on this, the analysis obtained a computed $\chi^{2}$ value of 13.412, with 6 degrees of freedom and a $\rho$-value of 0.037 , which suggest up to $95 \%$ chance that the uptake of IPTp significantly related to women's main source of health information.

\subsection{Support from Partners}

We requested participants to indicate if they received any form of support from their partners to facilitate their attendance of ANC clinics. The results indicated that 45 (37.2\%) participants who had obtained less than $\mathrm{IPTp}_{2}$ and $84(53.5 \%)$ who accessed $\mathrm{IPTp}_{2}+$ reported receiving support from their partners towards ANC visits. The analysis obtained a computed $\chi^{2}$ value of 6.671 (adjusted for continuity), with 1 degree of freedom and a $\rho$-value of 0.010 , which suggest up to $95 \%$ chance that the uptake of IPTp significantly associated with the availability of support from partners. In addition, participants received support from their partners in various forms with the most common being financial assistance, according to 28 (62.2\%) reporting less than $\mathrm{IPTp}_{2}$ and 57 (67.9\%) who had accessed $\mathrm{IPTp}_{2}+$. Besides, 12 (26.7\%) participants who had received less than $\mathrm{IPTp}_{2}$ and 14 (16.7\%) who indicated $\mathrm{IPTp}_{2}+$ acknowledged that their partners provided company during ANC visits; while other participants said their partners supported through reminders about dates for subsequent visits. However, the analysis revealed lack of significant relationship between the uptake of IPTp the form of support from partners (computed $\chi^{2}$ value $=2.594, \mathrm{df}=3$ and $\rho$-value $=0.458$ ).

\subsection{Perceived Susceptibility to Malaria Infection}

Even though all the 278 participants were aware of malaria risk during pregnancy, some of them believed that they were not susceptible to malaria infection. In this regard, Figure 4 shows that 80 (66.1\%) participants who had accessed less than $\mathrm{IPTp}_{2}$ and 106 (67.5\%) who reported $\mathrm{IPTp}_{2}+$ believed that they were susceptible to malaria infection. About one third in each group felt that they were not susceptible. Based on this, the analysis obtained a computed $\chi^{2}$ value of 0.014 (corrected for continuity), with 1 degree of freedom and a $\rho$-value of 0.907, suggesting that the relationship between the uptake of IPTp services and self-reported risk-perception was not significant. This is because most participants perceived themselves to be at risk of malaria infection, irrespective of whether they had received less than or full doses of IPTp.

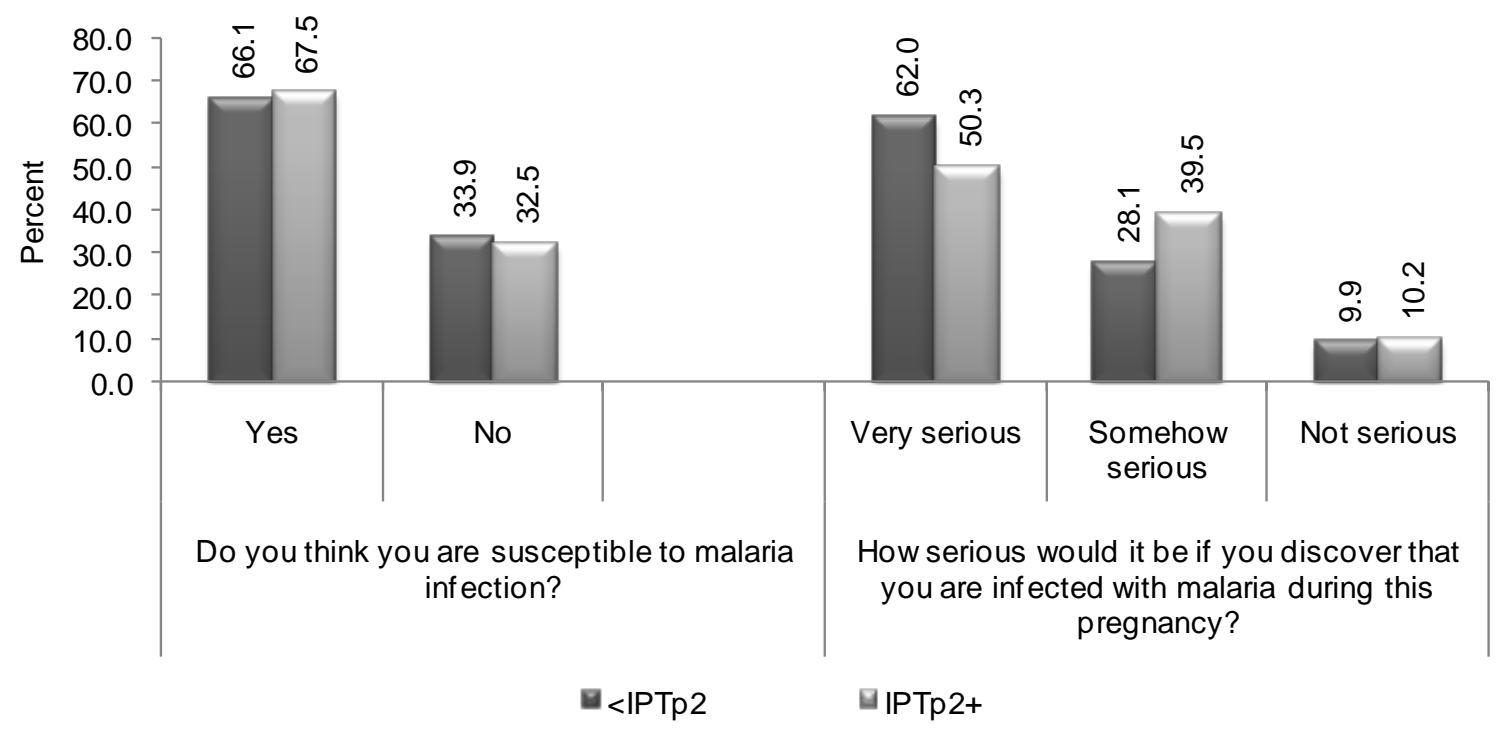

Figure 4. Risk perceptions and the seriousness of malaria infection

Figure 4 further shows that most participants perceived that malaria infection during the pregnancy would be a very serious or somehow serious matter. More particularly, up to $75(62.0 \%)$ participants who had received less than
$\mathrm{IPTp}_{2}$ and 79 (50.3\%) who had accessed $\mathrm{IPTp}_{2}+$ rated malaria infection during pregnancy as 'very serious', while $34(28.1 \%)$ who reported less than $\mathrm{IPTp}_{2}$ and 62 (39.5\%) who had received $\mathrm{IPTp}_{2}+$ felt that malaria 
infection during pregnancy would be 'somehow serious'. However, the analysis revealed that the relationship between the uptake of IPTp and perceived seriousness of malaria infection during pregnancy was not significant (computer $\chi^{2}$ value $=4.251, \mathrm{df}=2$ and $\rho$-value $=0.119$ ) Regardless of the IPTp doses, most participants demonstrated understanding that malaria infection during pregnancy was either a very serious or a somehow serious matter.

\subsection{Quality of Care at Health Facilities}

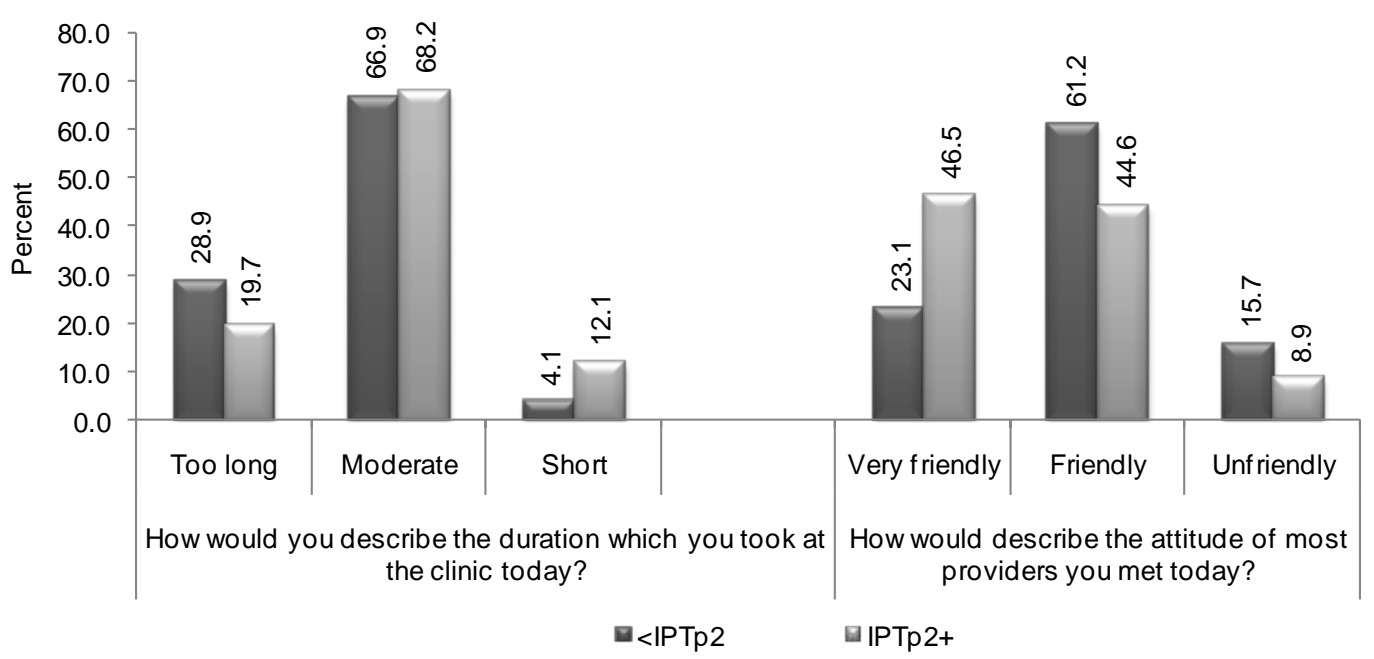

Figure 5. Perceptions about the quality of care at ANC facilities
More still, Figure 5 shows participants' perceptions regarding the attitude of most providers at the ANC facilities. In this regard, 28 (23.1\%) participants who had taken less than $\mathrm{IPTp}_{2}$ and 73 (46.5\%) who had obtained $\mathrm{IPTp}_{2}+$ described the attitude of most providers at the usual ANC facility as 'very friendly', while 19 (15.7\%) with less than $\mathrm{IPTp}_{2}$ and 14 (8.9\%) who reported $\mathrm{IPTp}_{2}+$ believed that most providers at the usual facility were 'very unfriendly'. The cross tabulation results suggested up to $99 \%$ chance that the uptake of IPTp significantly associated with perceived attitudes of providers towards pregnant women attending ANC clinics (computed $\chi^{2}$ value $=16.534, \mathrm{df}=2$ and $\rho$-value $=0.000)$.

\subsection{Factors Influencing the Uptake of Intermittent Preventive Treatment}

Bivariate analysis results in the preceding sub-sections indicated that the uptake of IPTp significantly associated with women's background attributes, including marital status, parity, education level, occupation and income level. The analysis also revealed significant relationships between the uptake of IPTp and independent variables such as the onset of ANC visits, distance to health facilities, partner support, perceptions about the duration at health facilities, providers' attitude and main source of health information.

To determine the influence of each variable on the uptake of IPTp, I incorporated the independent variables and the background profile factors in binary logistic regression model, using stepwise likelihood ratio method.
We requested participants to describe their perception about the duration they usually took at ANC clinics before (28.9\%) participants who received less than $\mathrm{IPTp}_{2}$ and 31 (19.7\%) who reported $\mathrm{IPTp}_{2}+$ described the duration they usually took at the facility as 'too long', while 81 (66.9\%) with less than $\mathrm{IPTp}_{2}$ and 107 (68.2\%) who received $\mathrm{IPTp}_{2}+$ felt that the duration was 'moderate'. Based on this, the analysis obtained a computed $\chi^{2}$ value of 7.468 , with 2 degrees of freedom and a $\rho$-value of 0.024 , suggesting up to $95 \%$ chance that the uptake of IPTp significantly associated with the duration taken at the health facilities before receiving services. receiving services. In this regard, Figure 5 shows that 35

The analysis process generated two models; with the first model having independent variables only and the second model adjusted to include background profile attributes. Table 3 provides summary results of the regression analysis. I have summarized the discussion in this subsection to focus on the results of the adjusted model and key findings only.

\subsubsection{Collinearity Diagnostics}

We tested the interrelationship between independent variables and background factors for collinearity indicators. Using the default outlier value of 2.0, we examined the standard errors (S.E.) associated with regression coefficients $(\beta)$. In this regard, standard errors larger than 2.0 indicated the existence of multicollinearity effects. We noted that the inclusion of marital status and parity inflated the standard errors, with parity status having a stronger effect. Based on this, I excluded parity status from the analysis. I noted the same challenge with average income and occupation, leading to exclusion of the latter from the analysis.

\subsubsection{Odds Ratios}

The results summarized in Table 4 suggest that women starting ANC visits in the second month had about 10.5 times the odds of taking $\mathrm{IPTp}_{2}+$ as those initiating ANC attendance in the sixth month of pregnancy ( $\rho$-value = $0.013, \beta=2.355$, C.I. $=3.16-35.18$ ). Those visiting ANC facilities for the first time in the third month had about 6.4 times the odds of taking IPTp $\mathrm{P}_{2}+$ as those starting at the sixth month $(\rho$-value $=0.022, \beta=1.854$, C.I. $=2.10-19.44)$. 
The results suggest that the earlier the onset of ANC visits the higher the likelihood of pregnant women taking $\mathrm{IPT}_{2}+$ the $24^{\text {th }}$ month of pregnancy.

The results in Table 4 further shows that women who cited radio as the main source of health information had about 3.7 times the odds of taking IPTp $\mathrm{P}_{2}+$ as those relying on community health workers ( $\rho$-value $=0.031, \beta=1.304$, C.I. = 1.79-7.58). However, those mentioning health facilities as the main source of information were about 12.7 times as likely to take $\mathrm{IPTp}_{2}+$ as who relied on community health workers ( $\rho$-value $=0.003, \beta=2.540$, C.I. $=6.91-21.10)$. The results suggest that health facilities were the most critical source of health information, thus, necessitating appropriate interventions to make health education programs attractive to women.
Table 4 shows that women who received support from their partners towards ANC visits were about 8.2 times as likely to take $\mathrm{IPTp}_{2}+$ as those who did not receive partner support $(\rho$-value $=0.005, \beta=2.105$, C.I. $=3.58-18.84)$. Based on this, partner support is critical for effective prevention of malaria in pregnancy. Furthermore, women dwelling within a distance of less than one hour's walk had about 6.4 times the odds of taking $\mathrm{IPTp}_{2}+$ as those dwelling within a distance of more than 4 hour's walk ( $\rho$ value $=0.039, \beta=1.852$, C.I. $=2.32-17.49$ ). The results suggest that the shorter the distance to ANC facilities, the higher the odds of women taking $\mathrm{IPTp}_{2}+$. Hence, distance to facilities emerged as a key factor influencing the IPTP $_{2}+$ uptake.

Table 4. Summary results of the adjusted logistic regression model

\begin{tabular}{|c|c|c|c|c|c|c|c|c|}
\hline \multirow{2}{*}{ Covariates } & \multirow{2}{*}{$\beta$} & \multirow{2}{*}{ S.E. } & \multirow{2}{*}{ Wald } & \multirow{2}{*}{ df } & \multirow{2}{*}{$\rho$-value } & \multirow{2}{*}{$\operatorname{Exp}(\beta)$} & \multicolumn{2}{|c|}{$95 \%$ C.I. for $\operatorname{EXP}(\beta)$} \\
\hline & & & & & & & Lower & Upper \\
\hline Onset of ANC visits & & & 17.264 & 4 & $0.006 * * *$ & & & \\
\hline 2nd month & 2.355 & 0.615 & 14.663 & 1 & $0.013^{* *}$ & 10.538 & 3.157 & 35.177 \\
\hline 3rd month & 1.854 & 0.568 & 10.654 & 1 & $0.022^{* *}$ & 6.385 & 2.097 & 19.439 \\
\hline 4th month & 0.965 & 0.398 & 5.879 & 1 & 0.225 & 2.625 & 1.203 & 5.726 \\
\hline 5th month & 0.352 & 0.180 & 3.824 & 1 & 0.249 & 1.422 & 0.999 & 2.023 \\
\hline 6th month + & 1.000 & 1.000 & 1.000 & 1.000 & 1.000 & 1.000 & 1.000 & 1.000 \\
\hline Main source of h/information & & & 13.446 & 6 & $0.028 * *$ & & & \\
\hline Radio & 1.304 & 0.368 & 12.556 & 1 & $0.031 * *$ & 3.684 & 1.791 & 7.578 \\
\hline Television & -1.474 & 1.098 & 1.802 & 1 & 0.206 & 0.229 & 0.027 & 1.97 \\
\hline H/facility & 2.540 & 0.600 & 17.921 & 1 & $0.003 * * *$ & 12.680 & 6.912 & 21.10 \\
\hline Friends & -1.712 & 0.716 & 5.717 & 1 & 0.296 & 0.181 & 0.044 & 0.734 \\
\hline Partner & -2.978 & 0.747 & 15.893 & 1 & $0.017 * *$ & 0.051 & 0.012 & 0.22 \\
\hline Community h/workers (RC) & 1.000 & 1.000 & 1.000 & 1.000 & 1.000 & 1.000 & 1.000 & 1.000 \\
\hline Partner support & & & 24.932 & 1 & $0.000 * * *$ & & & \\
\hline Yes & 2.105 & 0.424 & 24.647 & 1 & $0.005 * * *$ & 8.207 & 3.575 & 18.841 \\
\hline No (RC) & 1.000 & 1.000 & 1.000 & 1.000 & 1.000 & 1.000 & 1.000 & 1.000 \\
\hline Distance to the health facility & & & 14.817 & 3 & $0.021^{* *}$ & 1.000 & & \\
\hline$<1$ hour & 1.852 & 0.515 & 12.932 & 1 & $0.039 * *$ & 6.373 & 2.322 & 17.486 \\
\hline 1 to 2 hours & 0.938 & 0.316 & 8.811 & 1 & 0.314 & 2.555 & 1.375 & 4.746 \\
\hline 3 to 4 hours & -1.283 & 0.569 & 5.084 & 1 & 0.383 & 0.277 & 0.091 & 0.846 \\
\hline$>4$ hours $(\mathrm{RC})$ & 1.000 & 1.000 & 1.000 & 1.000 & 1.000 & 1.000 & 1.000 & 1.000 \\
\hline Waiting duration at h/facility & & & 16.999 & 2 & $0.019 * *$ & & & \\
\hline Short & 1.125 & 0.276 & 16.614 & 1 & $0.011^{* *}$ & 3.080 & 1.793 & 5.291 \\
\hline Moderate & 0.868 & 0.335 & 6.714 & 1 & 0.283 & 2.382 & 1.235 & 4.593 \\
\hline Too long (RC) & 1.000 & 1.000 & 1.000 & 1.000 & 1.000 & 1.000 & 1.000 & 1.000 \\
\hline Providers' attitude & & & 16.734 & 2 & $0.020 * *$ & & & \\
\hline Very friendly & 2.084 & 0.535 & 15.174 & 1 & $0.013^{* *}$ & 8.037 & 2.816 & 22.934 \\
\hline Friendly & 1.858 & 0.591 & 9.884 & 1 & $0.037 * *$ & 6.411 & 2.013 & 20.417 \\
\hline Unfriendly (RC) & 1.000 & 1.000 & 1.000 & 1.000 & 1.000 & 1.000 & 1.000 & 1.000 \\
\hline Marital status & & & 20.258 & 4 & $0.006 * * *$ & & & \\
\hline Single & 1.773 & 0.417 & 18.078 & 1 & $0.012^{* *}$ & 5.888 & 2.6 & 13.334 \\
\hline Married & 1.457 & 0.436 & 11.167 & 1 & $0.042 * *$ & 4.293 & 1.827 & 10.09 \\
\hline Separated & -0.230 & 0.470 & 0.239 & 1 & 0.135 & 0.795 & 0.316 & 1.996 \\
\hline Divorced & -0.522 & 0.465 & 1.260 & 1 & 0.247 & 0.593 & 0.238 & 1.476 \\
\hline Widowed (RC) & 1.000 & 1.000 & 1.000 & 1.000 & 1.000 & 1.000 & 1.000 & 1.000 \\
\hline Education level & & & 22.094 & 4 & $0.003 * * *$ & & & \\
\hline University & 1.404 & 0.302 & 21.613 & 1 & $0.007 * * *$ & 4.071 & 2.253 & 7.359 \\
\hline College & 1.099 & 0.312 & 12.408 & 1 & $0.036 * *$ & 3.001 & 1.628 & 5.532 \\
\hline Secondary & 0.789 & 0.427 & 3.414 & 1 & 0.166 & 2.201 & 0.953 & 5.083 \\
\hline Primary & -0.378 & 1.396 & 0.073 & 1 & 0.158 & 0.685 & 0.044 & 10.571 \\
\hline None (RC) & 1.000 & 1.000 & 1.000 & 1.000 & 1.000 & 1.000 & 1.000 & 1.000 \\
\hline Average income & & & 16.206 & 4 & $0.016 * *$ & & & \\
\hline KES 20,000+ & 1.208 & 0.304 & 15.790 & 1 & $0.018 * *$ & 3.347 & 1.844 & 6.073 \\
\hline KES 15,000-19,999 & 1.198 & 0.311 & 14.839 & 1 & $0.022 * *$ & 3.313 & 1.801 & 6.096 \\
\hline KES 10,000-14,999 & 1.069 & 0.774 & 1.908 & 1 & 0.158 & 2.912 & 0.639 & 13.277 \\
\hline KES 5,000-9,999 & 0.427 & 1.320 & 0.105 & 1 & 0.433 & 1.533 & 0.115 & 20.373 \\
\hline$<$ KES 5,000 & 1.000 & 1.000 & 1.000 & 1.000 & 1.000 & 1.000 & 1.000 & 1.000 \\
\hline Constant & 4.270 & 0.695 & 37.747 & 1 & $0.000 * * *$ & 71.522 & & \\
\hline
\end{tabular}

RC= Reference category; *, **, *** show significance at $0.1,0.05$ and 0.01 , error margins, respectively

The analysis further indicated women perceiving the duration of stay at health facilities before receiving services as 'short' were about 3.1 times as likely to take $\mathrm{IPTp}_{2}+$ as those saying that the duration taken was 'too long' $(\rho$-value $=0.011, \beta=1.125$, C.I. $=1.79-5.29)$. This implies that the longer the waiting time at health facilities the lower the odds of women taking $\mathrm{IPTp}_{2}+$ by the $24^{\text {th }}$ month of gestation. The results in Table 4 further show 
that women who perceived that most providers at the clinics were 'very friendly' had up to 8.0 times the odds of taking IPT $\mathrm{p}_{2}+$ as those hinting that most providers were 'unfriendly' ( $\rho$-value $=0.013, \beta=2.084$, C.I. $=2.82-$ 22.93). Hence, providers' attitude towards clients was a critical factor influencing malaria prevention among pregnant women.

More still, single women had about 5.9 times the odds of taking IPTp $2+$ as those widowed ( $\rho$-value $=0.012, \beta=$ 1.773 , C.I. $=2.60-13.33$ ). Besides, the results show women in marital unions had about 4.3 times the odds of taking $\mathrm{IPT}_{2}+$ as those widowed ( $\rho$-value $=0.042, \beta=$ 1.457 , C.I. $=1.83-10.09$ ). The results in Table 3 further indicate that women with university education had about 4.1 times the odds of taking $\mathrm{IPTp}_{2}+$ as those with no formal education ( $\rho$-value $=0.007, \beta=1.404$, C.I. $=2.25$ 7.36). Those having college qualifications were about 3.0 times as likely to take IPTp $\mathrm{P}_{2}+$ as those lacking forma education $(\rho$-value $=0.036, \beta=1.099$, C.I. $=1.63-5.53)$.

Furthermore, the analysis indicated women earning KES 20,000 or more had about 3.3 times the odds of taking IPTp $\mathrm{P}_{2}+$ as those earning less than KES 5,000 ( $\rho-$ value $=0.018, \beta=1.208$, C.I. $=1.84-6.07)$. Those in the KES 15,000-19,999 were about 3.3 times as likely to take IPTp $_{2}+$ as those in the bottom income bracket ( $\rho$-value $=$ $0.022, \beta=1.198$, C.I. $=1.80-6.10)$. The results suggest that the higher the average income the better the odds of women taking IPTp $\mathrm{P}_{2}+$ within the stipulated time.

\subsubsection{The Model's Goodness-of-fit}

We tested the goodness of this model using Nagelkerke's $\mathrm{R}^{2}$ and Hosmer-Lemeshow (H-L) goodnessof-fit statistic. In this regard, the analysis obtained a Nagelkerke's $\mathrm{R}^{2}$ of 0.735 implying that the model predicted up to $73.5 \%$ of variance in $\mathrm{IPT}_{2}$ uptake; and suggesting a strong relationship between women's perceptions and $\mathrm{IPTp}_{2}$ uptake. The $\mathrm{H}-\mathrm{L}$ goodness-of-fit statistic shows that a logistic regression model is well fitting the observed data at an acceptable level when the resultant $\rho$-value is greater than 0.05 ; further indicating that the model prediction does not significantly differ from the observed frequencies. In this study, the $\mathrm{H}-\mathrm{L}$ table obtained a $\chi^{2}$ value of 2.808 , with 8 degrees of freedom and a p-value of 0.271 , which is higher than 0.05 . This result confirms that our model was a good fit. In addition, omnibus tests of model co-efficients obtained a computed $\chi^{2}$ value of 82.715 , with 33 degrees of freedom and a pvalue of 0.000 , which was significant at 0.01 error margin, confirming up to $99 \%$ chance that the model-fit was statistically significant.

\section{Conclusions and Recommendations}

The aim of this study was to identify and document factors influencing the uptake of IPTp with a view to justifying the need for appropriate interventions that would strengthen the Kenyan IPTp program towards the realization of the Roll Back Malaria targets. The success of IPTp programs at the facility level will feed into and contribute to the excellence of the national program, by reducing maternal, fetal and infant mortality trends due to malaria infection.
The onset of ANC visits is a critical factor influencing the uptake of IPTp in Bungoma East District. In this regard, women starting ANC visits in the second month had about 10.5 times the odds of taking $\mathrm{IPTp}_{2}+$ as those initiating ANC attendance in the sixth month of pregnancy $(\rho$-value $=0.013, \beta=2.355$, C.I. $=3.16-35.18)$. In view of this, continuous sensitization of pregnant women through a multi-media approach is a critical intervention in which the government and development partners should invest to encourage early and consistent attendance of ANC facilities. At the facility level, the Government should improve the programming and content of health education sessions, while minding the needs and circumstances of pregnant women from various local communities. Sensitization should also target the general community to enable them play an active role in ensuring that all pregnant women begin their ANC visits at least by the fourth month of gestation.

Health information is important in shaping knowledge, perceptions and attitudes to facilitate behavior change. The source of such information is critical for its internalization, acceptability and translation into action. In the study area, pregnant women obtained health information from various sources, including radio, television, friends and community health workers. However, information accessed from health facilities had the greatest impact in terms of IPTp $\mathrm{p}_{2}+$ uptake as indicated by the Wald statistic (Table 4). In this regard, women who mentioned health facilities as the main source of information were about 12.7 times as likely to take IPT $\mathrm{P}_{2}+$ as who relied on community health workers ( $\rho$-value $=$ $0.003, \beta=2.540$, C.I. $=6.91-21.10$ ).

This suggests that information provided by health facilities was bound to be clearer and more authoritative than information provided by community health workers, serving as volunteers with minimal training. This reemphasizes the need for the Government to improve the quality of health education program during clinic days, through better programming, adequate staffing, better facilities and materials, as well through staff motivation. Reaching out to development actors and humanitarian agencies for partnership arrangements is an option that the Government should consider to access supplementary resources to support health education at ANC facilities.

Partners have an important role to play in the realization of IPTp program targets. Their involvement is not only socially logical, but also and more importantly, a matter of women's rights. In Bungoma East District, women who received support from their partners towards ANC visits had about 8.2 times the odds of taking IPTp ${ }_{2}+$ as those who did not receive partner support ( $\rho$-value $=$ $0.005, \beta=2.105$, C.I. $=3.58-18.84)$. However, in most patriarchal communities, matters related to pregnancy form part of women's responsibility, alongside childcare, farm work and domestic chores. Too many roles during pregnancy may compromise the time and resources that would enable women to access ANC services in good time for $\mathrm{IPTp}_{2}+$ services. Thus, men have an immense responsibility to support women to facilitate early and consistent attendance of ANC clinics. The success of the IPTp program depends on the extent to which men are involved and targeted with information for better knowledge, understanding and attitude change. Thus, the government and development partners should consider 
investing in the sensitization of men through the mass media, work place paternal programs and peer education.

Distance to health facilities is also a critical factor influencing $\mathrm{IPTp}_{2}+$ uptake. Shortage of health facilities vis-à-vis increment in human population is the main factor contributing to the challenge of long distance between available facilities and residences. For poor households, coping with transportation costs remains a key challenge to early and consistent ANC attendance, which is an antecedent of the IPTp $\mathrm{P}_{2}+$ uptake. In the study area, women dwelling within a distance of less than one hour's walk had about 6.4 times the odds of taking $\mathrm{IPTp}_{2}+$ as those dwelling within a distance of more than 4 hour's walk ( $\rho$ value $=0.039, \beta=1.852$, C.I. $=2.32-17.49)$. Construction of new health facilities in high-risk malaria zones is an investment that the Central and Devolved Governments should prioritize to improve access, particularly to pregnant women from poor households. As a short-term measure, the Government should establish partnership arrangements with development partners to support the mobile clinics program in areas that are seriously constrained to improve the IPTp uptake.

The duration of taken in health facilities before receiving services is an element of quality of care that also influences the uptake of $\mathrm{IPTp}_{2}+$ services. In this regard, women perceiving the duration of stay at health facilities before receiving services as 'short' were about 3.1 times as likely to take IPTp ${ }_{2}+$ as those saying that the duration taken was 'too long' $(\rho$-value $=0.011, \beta=1.125$, C.I. $=$ 1.79-5.29). This factor connects to a number of human resource management issues, including understaffing, inadequate remuneration, poor work environment, ineffective supervisory system and lack of appropriate motivation programs. Of all resources in an institution, human resource is the most critical, because it plans and manages the utilization of other resources to achieve institutional goals. Consequently, the Government should recruit and deploy more health workers to rural health facilities to improve staffing level and improve staff motivation for better services to pregnant women. Improving staffing and staff welfare as well as supervisory systems are likely to improve the IPTp uptake.

The attitude of health workers towards clients is also an element of quality of care. In the study area, women who perceived that most providers at the clinics were 'very friendly' had up to 8.0 times the odds of taking $\mathrm{IPT}_{2}+$ as those hinting that most providers were 'unfriendly' ( $\rho$ value $=0.013, \beta=2.084$, C.I. $=2.82-22.93$ ). Negative attitudes arise from the human resource issues highlighted in the preceding paragraph, as well as lack of training in the practice of customer care. Many studies have recommended the need for staff training and retraining in customer care practices to improve attitudes. However, inadequate budgetary allocation to the health sector constrains implementation. Although training health workers in public health facilities may be a costly undertaking, bearing in mind other recurrent costs centres, the Government should consider establishing partnership initiatives with the private sector and development partners to training health workers in appropriate skills and improve the quality of care.

Among participants' personal attributes, education and income level emerged as the most critical for programmatic interventions. In view of these results, supporting women to initiate income-generating activities and keeping the girl child longer in school are key interventions that should go a long way in improving the uptake of IPTp services in Bungoma East District. Already the Government has initiated Women's Enterprise Development Fund and the free basic education to enhance access, issues of funding inadequacy and inconsistency, as well as institutional capacity, ethnicity, financial misappropriation and access to basic needs for the girl child remain key challenges. Detaching these initiatives from political influence, strengthening responsible institutions through legislation and involving partners are key options that the Government should consider to empower women, improve girl-child education, and by extension, improve the IPTp uptake in Kenya.

\section{Limitations of the Study}

The sample size that we used in this study was about one-tenth of the target population. However, the maximum likelihood estimation (MLE) method used in logistic regression relies on large-sample asymptotic normality, implying that the reliability of estimates decline when cases for each observed independent variable are too few. We coped with this limitation by excluding some background profile variables from the analysis and merging some variable categories. Future studies should expand the scope of variables and sample size to generate better regression models of factors influencing the IPTp uptake.

\section{Acknowledgement}

I thank all the institutions and individuals who supported this study through ideas, information and logistics. I am grateful to Moi University for granting me opportunity to pursue the Master of Public Health Degree. I acknowledge the support and guidance of my supervisors Prof. Misia Kadenyi and Dr. Joyce Baliddawa. I thank my research assistants for supporting data collection led by Eric Ernest Mangu'unyi a Research Fellow at Catholic University of Eastern Africa, and Mr. Tom Odhiambo, a lecturer at the University of Nairobi, for data processing and analysis. Finally, I am deeply indebted to all participants who volunteered their time, personal information, knowledge and experiences, which form the substance of this paper.

\section{References}

[1] World Health Organization (2010). World Malaria Report 2010. Geneva: Global Malaria Program.

[2] World Health Organization (2011). World Malaria Report 2011. Geneva: Global Malaria Program.

[3] World Health Organization (2012a). World Malaria Report 2012. Geneva: Global Malaria Program.

[4] Kenya National Bureau of Statistics and ICF Macro (2010). Kenya Demographic and Health Survey 2008-09. Calverton, Maryland: KNBS and ICF Macro.

[5] Ministry of Public Health \& Sanitation (2009). National Malaria Strategy 2009-2017. Nairobi.

[6] Steketee, R.W., Wirima, J.J., Campbell, C.C. "Developing effective Strategies for Malaria Prevention Programs for Pregnant African Women.” Am J of Trop Med and Hyg1996; 55:95-100. 
[7] Luxemburger, C., Ricci, F., Nosten, F., Raimond, D., Bathet, S. and White, N. J. "The Epidemiology of Severe Malaria in an Area of Low Transmission in Thailand.” Transactions of the Royal Society of Tropical Medicine and Hygiene 1997; 91:256-262.

[8] World Health Organization (2007). Malaria in Pregnancy: Guidelines for Measuring Key Monitoring and Evaluation Indicators. Geneva: Global Malaria Program.

[9] World Health Organization (2005). A strategic framework for malaria prevention and control during pregnancy in the African Region. Brazzaville: WHO.

[10] Ministry of Public Health and Sanitation, Kenya National Bureau of Statistics and ICF Macro (2011). 2010 Kenya Malaria Indicator Survey. Nairobi: DOMC, KNBS and ICF Macro.

[11] Ouma, P.O., van Eijk, A.M., Hamel, M.J., Sikuku, E., Odhiambo, F., Munguti, K., Ayisi, J.G., Kager, P.A. and Slutsker, L. "The Effect of Health Care Worker Training on the Use of Intermittent Preventive Treatment for Malaria in Pregnancy in Rural Western Kenya”. Tropical Medicine and International Health 2007; 12(8): 953-961.

[12] Mpungu, S. K. and Mufubenga, P. "Use of Antenatal Care, Maternity Services, Intermittent Presumptive Treatment and Insecticide Treated Bed Nets by Pregnant Women in Luwero District, Uganda.” Malaria Journal 2008; 7: 44.

[13] Marchant, T., Nathan, R. Jones, C., et al. "Individual, Facility and Policy Level Influences on National Coverage Estimates for Intermittent Preventive Treatment of Malaria in Pregnancy in Tanzania”. Malaria Journal 2008; 7(1): 260.

[14] Olliaro, P.L, Delenne, H., Cisse, M., Badiane, M. Olliaro, A., Vaillant, M. and Brasseur, P. "Implementation of Intermittent Preventive Treatment in Pregnancy with SulphadoxinePyrimethamine at a District Health Centre in Rural Senegal”. Malaria Journal 2008; 7: 234.

[15] van Eijk, A.M., Ayisi, J.G., ter Kuile, F.O., Slutsker, L., Otieno, J.A., Misore, A.O., Odondi, J.O., Rosen, D.H., Kager, P.A., Steketee, R.W., Nahlen, B.L. "Implementation of Intermittent Preventive Treatment with Sulphadoxine-Pyrimethamine for Control of Malaria in Pregnancy in Kisumu, Western Kenya”. Trop Med and Inter Health 2004; 9(5): 630-37.

[16] Anders, K., Marchant, T., Chambo, P., Mapunda, P. and Reyburn, H. "Timing of Intermittent Preventive Treatment for Malaria during Pregnancy and the Implications of Current Policy on Early Uptake in North-East Tanzania.” Malaria Journal 2008; 7:79.

[17] Nankwanga, H.A. and Gorette, N. "Adherence to Intermittent Preventive Treatment for Malaria in Pregnancy." Afri J of Midwifery and Women's Health 2008; 2:131-40.

[18] Nganda, R.Y., Drakeley, C., Reyburn, H., Marchant, T. "Knowledge of Malaria Influences the Use of Insecticide Treated Nets but Not Intermittent Presumptive Treatment by Pregnant Women in Tanzania.” Malaria Journal 2004; 12(3): 42.

[19] Enato, E.F., Okhamafe, A.O., Okpere, E.E. "A Survey of Knowledge, Attitude and Practice of Malaria Management among Pregnant Women from Two Health Care Facilities in Nigeria." Intern J Obst and Gyn 2007; 86(1): 33-36.

[20] Mubyazi, G., Bloch, P., Kamugisha, M., Kitua, A., Ijumba, J. "Intermittent Preventive Treatment for Malaria during Pregnancy: A Qualitative Study of Knowledge, Attitudes and Practices of District Health Managers, Antenatal Care Staff and Pregnant Women in Korogwe District, North-Eastern Tanzania.” Malaria Journal 2005; 20 (4):31.

[21] Tarimo, S.D. (2007). "Appraisal on the Prevalence of Malaria and Anaemia in Pregnancy and Factors Influencing Uptake of Intermittent Preventive Therapy with Sulfadoxine-Pyrimethamine in Kibaha District, Tanzania”. East African Journal of Public Health 2007; 4:80-3.

[22] Hill, J. and Kazembe, P. "Reaching the Abuja Target for Intermittent Preventive Treatment of Malaria in Pregnancy in African Women: A Review of Progress and Operational
Challenges.” Tropical Medicine and International Health 2006; 11(4):409-18.

[23] Mboera, E.G.L., Makundi, E.A. and Kitua, A.Y. "Uncertainty in Malaria Control in Tanzania: Crossroads and Challenges for Future Interventions”. American Journal of Tropical Medicine 2007; 77(6): 112-18.

[24] Ministry of Planning \& National Development (2010). Bungoma East District Annual Operational Plan 2010-2011. Nairobi.

[25] Hochbaum, G.M. (1958). Public Participation in Medical Screening Programmes: A Socio-Psychological Study. Washington DC: Government Printing Office.

[26] Glanz, K., Lewis, E.M. and Rimer, B.K. (2002). Health Behaviour and Health Education: Theory, Research and Practice. San Francisco: Jossey-Bass Publishers.

[27] McCormick-Brown, K. (1999). Health Belief Model. http://hsc.usf.ed/Health_Belief_Model_Overview.htm. Accessed on $22 / 4 / 2013$.

[28] Belcher, L. "Condom Use and Perceived Risk of HIV Transmission among Sexually Active Men”. AIDS Education and Prevention 2005; 17(1):79-89.

[29] Janz, N.K. and Becker, M.H. "The Health Belief Model: A Decade Later”. Health Education Quarterly 1984, 11(1):1-47

[30] Stretcher, V. and Rosenstock, I.M. (1997). The Health Belief Model. In Glanz, K., Lewis, F.M. and Rimer, B.K., (Eds.). Health Behaviour and Health Education: Theory, Research and Practice. San Francisco: Jossey-Bass.

[31] Ali, N.S. "Prediction of Coronary Heart Disease Preventive Behaviours in Women: A Test of the Health Belief Model". Women and Health 2002; 35(1): 83-96.

[32] Bandura, A. (1977). Social Learning Theory. Englewood Cliffs, New Jersey: Prentice-Hall Inc., 1977.

[33] Galloway, R.D. (2003). "Health Promotion: Causes, Beliefs and Measurements.” Clinical Medicine and Research 2003; 1(3):249258.

[34] Pender, N. J. (1996). Health Promotion in Nursing Practice ( $3^{\text {rd }}$ ed.). Connecticut: Appleton \& Lange Stanford.

[35] Peterson, S. J. and Bredow, T.S. (2009). Middle Range Theories: Application to Nursing Research $\left(2^{\text {nd }}\right.$ ed.). Philadelphia, PA: Lippincott, Williams \& Wilkins.

[36] Pender, N. (2011). The Health Promotion Model Manual. http://deepbluelib.umich.edu/bitstream/2027.42/85350/1/heal Accessed on 29th May 2013.

[37] Reed, P. and Shearer, N. (2009). Perspectives on Nursing Theory ( $5^{\text {th }}$ ed). Philadelphia, PA: Lippincott Williams \& Wilkins.

[38] McEwen, M. and Wills, E. (2011). Theoretical Basis for Nursing $\left(3^{\text {rd }}\right.$ ed.). Philadelphia, PA: Lippincott Williams \& Wilkins.

[39] KNBS (2010). The National Population and Housing Census 2009 Report, Nairobi, Kenya.

[40] Nachmias, C.F and Nachmias, D. (1996). Research Methods in the Social Sciences, $5^{\text {th }}$ Edition. London: Arnold.

[41] Berthoud, R. (2000). A measure of changing health. In Berthoud, $R$. and Gershuny (eds.), Seven years in the lives of British families: Evidence on the dynamics of social change from the British household panel survey. Bristol, UK: Policy Press.

[42] Westergaard, J., Noble, I. and Walker, A. (1989). After redundancy: The experience of economic insecurity. Cambridge, UK: Polity.

[43] Bryman, A. and Cramer, D. (1997). Quantitative Data Analysis with SPSS for Windows: a guide for Social Scientists. London: Routledge.

[44] Wuensch, K.L. (2006). Logistic Regression with SPSS. http://core.ecu.edu/psyc/wuenschk/spss/logistic.sav. Accessed on 22/4/2013.

[45] Rindfleisch, A., Malter, A.J., Ganesan, S. and Moorman, C. "Cross-Sectional Versus Longitudinal Survey Research.” Journal of Marketing Research 2008; 45(3): 1-23. 